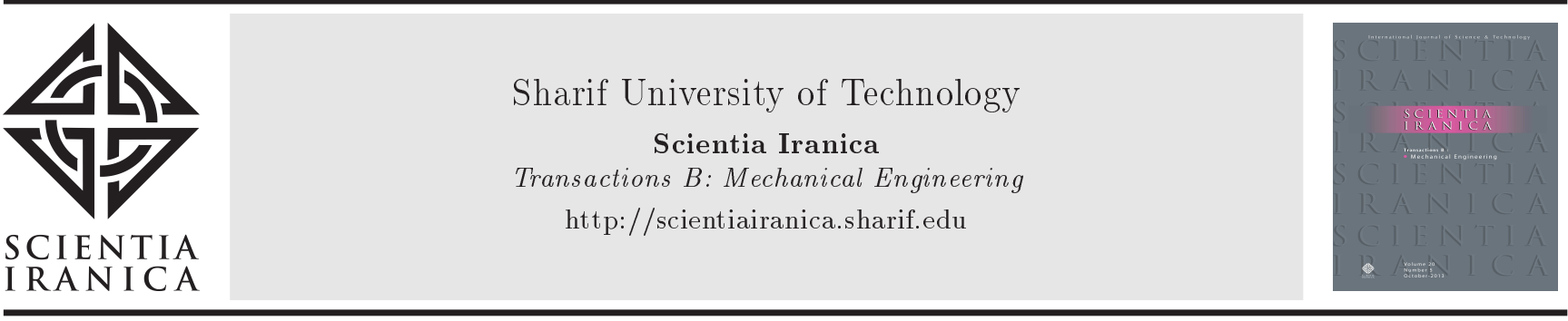

\title{
Drivers of cross-country vehicles
}

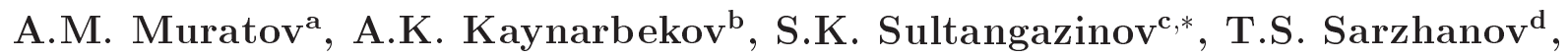 \\ A.K. Kazhigulova ${ }^{a}$ A. Shalkarov ${ }^{\mathrm{e}}$, G.S. Mussaeva ${ }^{\mathrm{f}}$, K.M. Bekmambet ${ }^{\mathrm{a}}$, \\ B.Sh. Yessengarayev ${ }^{g}$, and A. Tanirbergenov ${ }^{h}$
}

a. Department of the Transport Technology, Mechanical Engineering and Standardization, Kazakh University of Railway Transport, Kazakhstan, Almaty City, Zhetysu Street, 1 microdistrict, 73, 19.

b. Department of the Radio Engineering, Electronics and Telecommunications, Kazakh University of Railway Transport, Kazakhstan, Almaty City, Taugul Street, 1 microdistrict, 58, 5.

c. Department of the Automation and Transport Information Systems, Kazakh University of Railway Transport, Kazakhstan, Almaty City, Orbita Street, 2 microdistrict, 12, 36.

d. Department of the Transport Economics, Kazakh University of Railway Transport, Kazakhstan, Almaty City, Zhambyla Street, $93 A, 49$.

e. Department of the Transport Construction, Bridges and Tunnels, Kazakh University of Railway Transport, Kazakhstan, Almaty City, Tau Samaly Microdistrict, Tole bi Street, 9.

f. Department of the Transport Construction, Kazakh University of Railway Transport, Kazakhstan, Almaty City, Kurmangazy Street, $145 \mathrm{~A}, 3$.

g. Department of the Automation and Transport Information Systems, Kazakh University of Railway Transport, Kazakhstan, Kyzylorda Region, Kyzylorda City, Baitursynova Street, 18.

h. Department of the Railway Track, Railway Location Survey and Design, Kazakh University of Railway Transport, Kazakhstan, Aktobe City, Zarechny Street, Lane 1, 19.

Received 13 April 2018; received in revised form 19 July 2018; accepted 22 October 2018

\section{KEYWORDS}

Drivers;

Chassis;

Transport of rescuers;

Unmanned research

mechanisms;

Wheel-track.

\begin{abstract}
This work offers a new chassis design, namely the wheel-track, presents a mathematical model of this chassis, and also proves the advantage of the proposed design when driving the vehicle on arbitrary terrain, a rough off-road. The proposed approach is applied to design unmanned research mechanisms for other planets (Mars rovers, lunar rovers, etc.) and, also, to design robots and transport of rescuers at liquidation of consequences of natural or technological disasters. This article analyzes the requirements of the chassis design of the extraterrestrial unmanned research mechanism and demonstrates the high compliance of the proposed approach with results. This study proposes and investigates a mathematical model of wheel-track, demonstrates the optimization of the proposed mathematical model to machine computing, and shows the flexibility and scalability of the mathematical model. The proposed design is an attempt to combine the advantages of walking and wheel types of travel through one mechanism.

(C) 2019 Sharif University of Technology. All rights reserved.
\end{abstract}

\section{Introduction}

Humankind has created many machines and mecha-

*. Corresponding author.

E-mail address: suleke.kzsh@gmail.com (S.K. Sultangazinov)

doi: $10.24200 /$ sci. 2018.50781 .1862 nisms in an attempt to solve transportation problems. In most cases, wheel transport gets the job done on the Earth's surface and, even, on the surface of other planets. However, wheel transport is not universal, which raises this question: what can surpass wheel transport? The answer is movement with the help of legs, since many animal species move with the help of legs across terrain, where no wheel mechanism can traverse. What makes leg movement universal? Firstly, 
consider the wheel and the boundaries of its applicability. The limits of the applicability of the wheel lie in its interaction with the ground during movement across a surface that differs from the solid road surface; when moving across agricultural land, sand, snow, etc., the wheel loses its superiority [1]. It is necessary to highlight several key moments. On an unsolid surface, the wheel causes deformation of the ground surface that will increase during the rotational motion of the wheel, which is necessary for the vehicle to move forward, i.e., inevitable rotational motion. Secondly, solid wheel disks are used only in rail transport, while the soft rubber of pneumatic tires does not transfer pressure from the base to the wheel evenly. Simple model simulations show that the response reactions of the base that act from the surface onto the wheel are distributed unevenly across the "wheel-surface" contact patch: The response of the base at the center of the contact patch is at its maximum and tends to zero at the edge of the contact patch. This uneven distribution of forces exacerbates the deformation of the ground surface, thus weakening adherence. All these negative factors will only exacerbate during the rotational motion of the wheel.

Figure 1 shows the scheme of a tire with straight lugs in the elastic operating mode. The ground surface puts pressure on both the tread pattern and the tire body between the lugs of the tread pattern. The pressure of the ground surface of the tread pattern and the tire body can be described using the following equations:

$$
\begin{aligned}
& P_{t}=\left(\left(k_{c / b}+k_{\varphi}\right) z_{L}^{n},\right. \\
& P_{c r}=\left(\left(k_{c / b}\right)+k_{\varphi}\right)\left(z-h_{L}\right)^{n} .
\end{aligned}
$$

How are the forces distributed under the bearing surface of the foot in case of leg movement? A flat foot, when stepping on the ground surface in a downward vertical direction, will have an even distribution of forces under its surface even in motion, i.e., the stepbased movement will not deform the ground, which can be regarded as the key to steady travel across unsolid substrates.

There have been many projects of "walkers" (vehicles that move on legs); however, none of them managed to get to batch production. Apparently, multi-hinge mechanisms are too complicated for modern engineering. How can one combine the advantages of a stepping propelling device with simplicity and constructability that are required for batch production? There is a widely reproduced variant - the tracktype propelling device, which is used in military and agricultural machinery. The tracks are rigid; hence, they provide an even distribution of response forces of the base under their surface; in addition, unlike the wheel, they drop onto the substrate surface without sliding. The popularity of the track-type propelling device is well deserved, but such devices are massive. Light polymeric tracks could solve the weight problem (and weight-related problems of drag loss and loss due to rerolling of massive tracks); however, when turning, a track-type vehicle faces problems of substrate deformation - the need to turn a vehicle that rests on two rails of elastic track bands requires substrate deformation, while when moving on a solid surface, mechanical strain can cause early wear or even deformation of the track-type propelling device. Nevertheless, the need to ensure the maneuverability of vehicles forces inventors to create complex designs based on wheels; for example, Abdelrahman et al. [2] used the Mecanum wheel to ensure the maneuverability of the vehicle; however, this wheel type, while providing exceptional maneuverability on a solid surface, is unsuitable for off-road conditions. Giurgiu et al. [3], among other things, presented the analysis and statistics of damage that results from contact with the substrate surface, especially in case of an irregular surface, and discussed the highly required materials.

Thus, an ideal vehicle for off-road conditions should be a mechanism that incorporates the simplicity

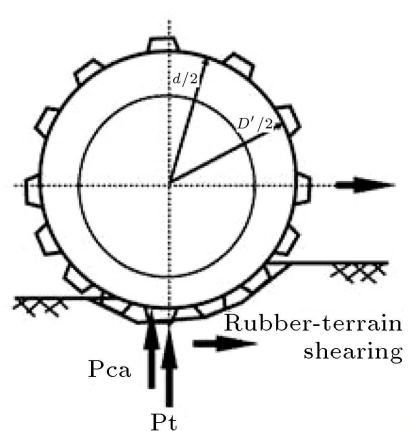

(a)

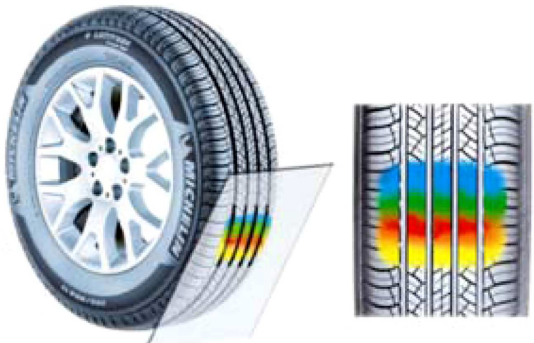

(b)

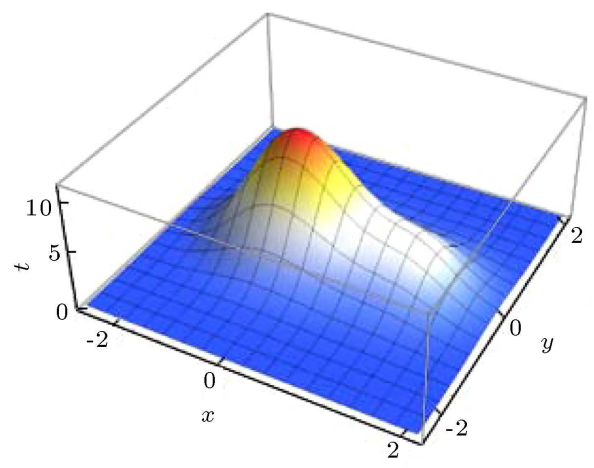

(c)

Figure 1. (a) A tire with lugs in the elastic operating mode. (b) Distribution of forces in the contact patch-ground surface. (c) Distribution of forces in the contact patch-wheel. 
of the wheel and a solid bearing surface that steps on the substrate without tangential displacements and ensures high maneuverability when compared to the track-type propelling device.

At the moment, difficult conditions of roadless areas encourage designers to experiment with the number of wheels, suspension, etc. using a well-known concept of the wheel [4]. However, in the majority of cases, attempts have been made to improve the suspension, but not the wheels (see, e.g. [5-7]). Meanwhile, many of the problems of the Mars rovers were concerned with the wheels.

Another important aspect is the distribution of forces during turning. Ideally, the wheel should transform and change its shape in proportion to the lateral response force. This deformation rolls the tire towards its longitudinal direction at the angle of its lateral slip. However, in this case, the term "slip" does not exactly imply slipping, but rather a deformation, during which the tire "goes" into the outer radius of the turn. In this case, maximum adherence with the surface will be achieved at a lateral angle size of more than zero.

It has been many centuries since the invention of the wheel, and mankind has not stopped attempting to improve it. Therefore, our work is not unique in this series. As an example of ongoing attempts to improve and "reinvent" the wheel, we can refer the readers to the design projects in $[8,9]$.

Given projects $[8,9]$ are just designs in their purest form. Unfortunately, a pretty picture does not contain necessary calculations; the mathematical model of the concepts and the behavior of the proposed projects on the surface are unclear, especially on the off-roads - we can only make conjectures in this regard. Meanwhile, on the surface of other planets that require the use of research machines, there is off-road in the extreme phase.

On this complex surface composed of loose silty sands as the outputs of shattered sedimentary rocks with the inclusion of relatively hard pebbles, it is impossible to move straightforwardly, and there is no need to move fast. Therefore, the approach of this research machine is forced winding, especially if it meets the outcrops on its way, which it will need to come around, or breeds that are of interest to the study, which it will have to approach for sampling and measurements in situ (Figure 2).

\section{Results and discussion}

Recently, in the press, the design of the walking wheel began to emerge based on the process of interaction of wheel elements with irregularities of homogeneous reference surface [10]. The geometric parameters of the walking wheel are determined based on the conditions of interaction with frontal obstacles of the soil.

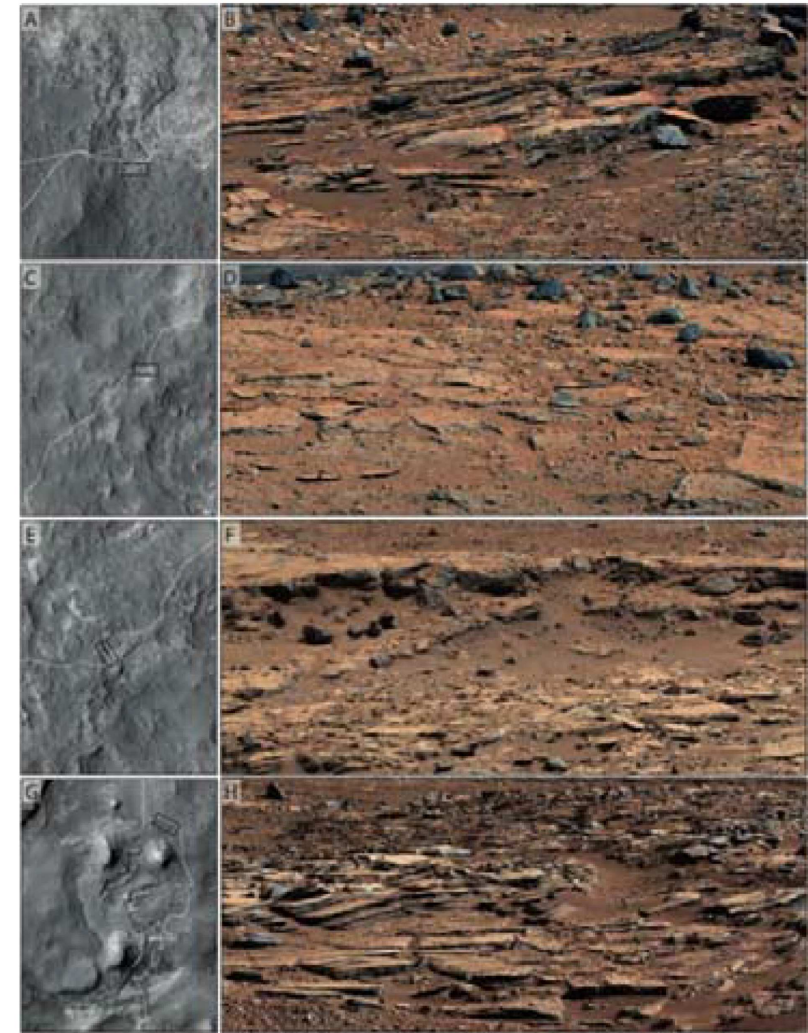

Figure 2. A winding road near the outcrops, where the traces of water flow were found presumably [10].

The advantage of these walking wheels equipped with curved feet is that riding it excludes the impact of the rim of the front obstacles of the soil. This is achieved by removing part of the rim of the round wheels. Thus, the obtained walking wheel is very simple in design (one piece made of the same material), is easy to move, rolls into the off-road conditions, and has a flotation of high relief on hard ground. Concerning walking wheels, the authors of [10-21] developed methods for the synthesis and analysis of the scheme of the walking wheel and four varieties that they investigated in layout. A common drawback of the walking wheel is considered to be the drop of the coming wheel feet from the height of $7-10 \mathrm{~mm}$ (the wheel parameter is $D=1200 \mathrm{~mm}$ ) on the supporting surface. The height of the drop, though not large in magnitude, creates discomfort when riding on the asphalt road; at high speeds, it creates vibration.

When riding on the off-road, the defect of the drive wheels becomes invisible.

The last modification of the walking wheel is made that consists of two elements, shin-foot and hip, which are connected by a hinge with limited movement relative to each other and connected through a damping elastic element [11].

The amount of the relative rotation of shin and hip in the knee joint is of the order of 40-60.

In this way, the walking wheel, "Kanbak" (Salsola 
paulsenii in Kazakh), is stable on a supporting surface, does not destroy it, and saves the straightness of the movement of the wheel hub with soft damping.

Thus, the authors of [10-21] believed to have solved the problematic task of creating a generic driver for motor vehicles, which can be used instead of pneuma-wheel when driving on the asphalt road inside the city, such that one can go for a walk on the off-road.

The subject of a universal driver, i.e., the walking wheel, was brought up earlier, which is intended for driving on a firm supporting surface with obstacles in the form of projections or pits, the height of which does not exceed the value of the radius of the wheel.

Off-roads are different. They can appear in various forms: nomadic sand, loose snowbanks, swamps (temporary wetlands and natural), rock blockages, mountain valleys, ridges, and gorges. In any case, the reference surface (soil) and geometric shape of the foot of the vehicle interact while riding.

The task of this paper is to pick up their options when designing a musculoskeletal system of the vehicle so that the machine can move without stopping under the action of the driving torque of the engine. For this purpose, at the beginning of the design, we should establish or select features of the reference surface. These characteristics are the permissible values of specific pressure of environment and friction coefficient between the support surface and environment, $[p]$ and $\left[f_{0}\right]$.

Based on these interactive parameters, the desired shape and parameter values of the musculoskeletal system should be determined, provided that the movement exists. These parameters are as follows:

$$
[p]=\frac{G}{W}\left(\frac{N}{\mathrm{~cm}^{2}}\right),
$$

where $[p]$ is the allowable specific pressure of the material of support surface $\left(\frac{N}{\mathrm{~cm}^{2}}\right), G$ is the total weight of the machine falling on one support apparatus (wheel), $W$ is the contact area of the foot of musculoskeletal apparatus $\left(\mathrm{cm}^{2}\right)$; in this respect, $[p]$ is given in the beginning of the design as an environmental parameter, $G$ is part of the machine weight falling on one musculoskeletal apparatus (specified parameter), and $W$ is the determined parameter of the foot, contact area of the foot.

$$
F_{m p}=f_{0} \cdot p \cdot W(N),
$$

where $F_{m p}$ is the friction force between the contacting elements $(N), f_{0}$ is the coefficient of friction between the materials of the contacting elements (dimensionless), and $G=N$ is the pressure or reaction of the support $(N)$.

The value of determined interacting elements must ensure the movement of the center of the wheel hub towards the reference surface:

$$
M_{g} \geq M_{\text {res }},
$$

where $M_{\text {res }}=F_{m p} . r ; r$ is the radius of the wheel (moment of resistance, acting towards the wheel contact N.m), and $M_{g}$ is the torsion torque acting on the wheel from the driver side (N.m).

According to Eq. (3), the musculoskeletal system moves the wheel hubs towards the support surface; if $M_{d r}-M_{r e s}>0$, the center of the wheel hub will move rapidly, i.e., there is acceleration of the wheel; if $M_{d r}-M_{r e s}=0$, the center of the wheel hub will move uniformly, i.e., there will be an established regime of movement; if $M_{r e s}>M_{g}$, there will be a slowdown, i.e., coasting.

The support surface is pliable, on which one is forced to travel by vehicles due to life circumstances, the mechanical properties of which are similar to several characteristics. For example, snow, sand, swamp, and water have weak internal communications, each resisting in their own way, but with similar forces. It is the permissible resistance, $[p]$, of the environment to a vertically acting load. One can hardly find in dictionaries the exact value of this parameter for each case in different places.

Therefore, while designing, the exact value of the specific pressure, [p], should be determined, at least in laboratory conditions. In addition, when designing structures, it is important to consider the accurate value of the coefficient of friction, $[f]$, for materials of supporting surface and foot of the musculoskeletal apparatus of the machine. Knowing $[p]$ and $[f]$ with a known weight, $G(N)$, one can determine the value of the area, $W$, of the contact support surface of the musculoskeletal apparatus $\left(\mathrm{cm}^{2}\right)$ :

$$
W=\frac{G}{[p]}\left(\mathrm{cm}^{2}\right),
$$

where $G$ is the weight falling on one foot (average).

In addition to weight, $\mathrm{G}$, on the support surface, the projection of $P_{d r}$ is applied, which is the driving force of the actuator and must be balanced by a friction force, $F_{f r}$, i.e.:

$$
P_{d r}-F_{f r}=0 .
$$

Only under these conditions there will be the movement of the center of the wheel hub of the vehicle. $F_{f r}$ equals:

$$
F_{f r}=\frac{G}{[f]} ;(G=N),
$$

where $N$ is the normal reaction support surface $(N)$.

Under these conditions, the design of parameters of the musculoskeletal apparatus of the machine is created.

Basically, the ingenuity of the designer manifests itself when creating a structural diagram of the musculoskeletal system, because there can be quite a lot of the varieties of designs. Below, one design of the 


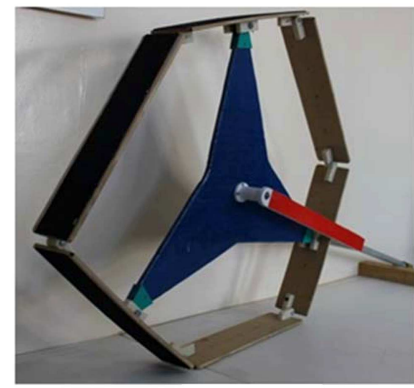

(a)

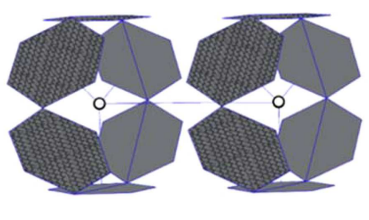

(b)
Figure 3. Photo of maqette of the wheel: (a) Track and (b) 3D model of the proposed wheel-track.

musculoskeletal system is presented, which is called the walking wheel-track and is designed to drive on a highly challenging support surface.

Figure 3 shows the current model of the wheeltrack that consists of tracks, which are closed with its links around the traffic generator of triangular shape and are pivotally connected with the generator in three points. The traffic generator is coaxially and pivotally connected with the drive shaft of the actuator. The track links are made as a foot with a calculated reference area; they are rectangular in shape and are interconnected pivotally by means of rods, forming a hexagonal shape.

Figure 4 shows a diagram of the walking wheeltrack with a sync ratchet device. The wheel-track contains six equal parts: caterpillar, covering the outside area of the traffic generator of triangular shape, which in the middle part at three points is pivotally connected with the covering elements of a caterpillar by fingers. This forms a strictly symmetrical system of three four-bar mechanisms, a ratchet disk, having six teeth coaxially and fixedly connecting in the disk center with traffic generator, clutch, and rotating on an axis fixed to drum of the drive shaft of the driver of the motor vehicle.

As shown in Figure 4, the mechanism of the wheeltrack is composed of the following: The shaft of motor is rigidly associated with drum, connected to a ratchet disk that is assembled with the traffic generator, and is fixed towards the drive shaft with a possibility of free rotation. Clutch is worn on the finger of drum and is also fixed with a possibility of free rotation; the other end is pulled by a spring to the drum so that the first shoulder of clutch is pulled against the teeth of the ratchet disk. In this respect, the wheel-track is set at one end of the drive shaft of the engine in the form of a wheel of one-way traffic. On the other end of the drive shaft of the engine, similarly, we set the second wheeltrack. As a result, the mechanism of the drive axle of the vehicle is formed, which has both wheel-tracks that rotate together with the drive shaft of the engine only forward in the direction of movement as a pair of wheels of railway carriages.

If we rotate the housing of the axle together with the drive shaft on the plane of the support surface, one wheel of the caterpillar stops, and the other rotates towards the stopped wheel-track with a speed rate twice the angular speed as the differential drive axle.

Such a device is structurally simple and provides precise turning of the vehicle securely by the rectilinear motion of the chassis, which is not susceptible to all sorts of drifts in the direction of slippery roads. Figure 5 shows a structural diagram of a vehicle equipped with four wheel-tracks of which two are leading and rotate synchronously as a pair of wheels, the other two are driven, and they are installed at the ends of the horizontal axis to manage a cross-shaped rear axle. The chassis comprises a frame (Figure 5(b)); front drive axle (shown in Figure 5 with number of 2 ) on the drive shaft of which two wheel-tracks are mounted that rotate together with the drive shaft synchronously through a six-tooth ratchet clutch; and the cruciform rear axle (shown in Figure 5 with number of 3 ) connected by its vertical stand with the common frame (Figure $5(\mathrm{~b})$ ) with a possibility to rotate relative to the total frame on a plane supporting surface. At the end of the horizontal axis of the cross-shaped axle, two wheeltracks (shown in Figure 5 with the numbers of 3 and 4 ) are pivotally mounted. The vehicle operates as follows:
1. Caterpillar

2. Traffic generator

3. Finger

4. Ratchet disk

5. Clutch

6. Drum

7. Motor

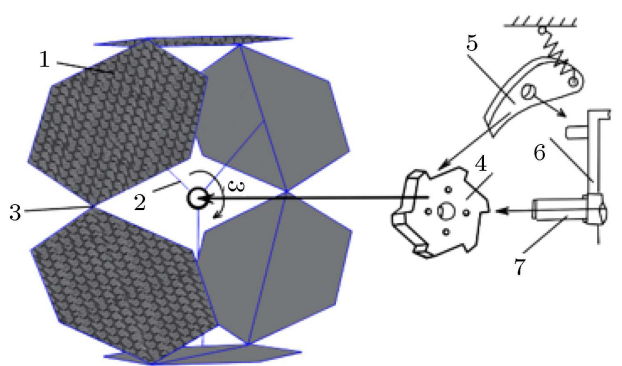

(a)

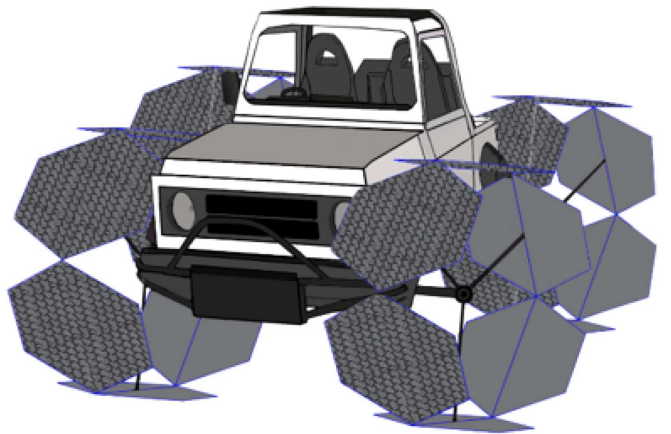

(b)

Figure 4. (a) The scheme of walking wheel-track with a synchronizing ratchet device. (b) Scheme of the vehicle with the chassis of the type wheel-track. 


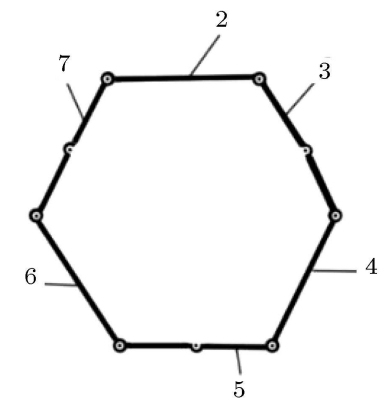

(a)

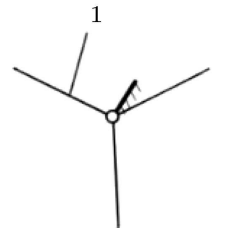

(b)

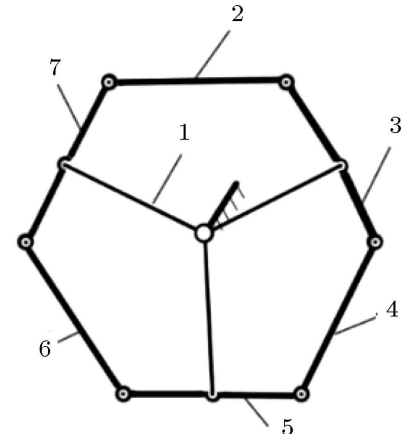

(c)

1: Triangular leading link generator;

$2,3,4,5,6,7$ : Support elements of equal size, which are interconnected pivotally, forming a closed planar kinematic chain.

Figure 5. Structural diagram of the wheel-track.

the movement of the actuator (motor) is transmitted via the transmission mechanism to the drive shaft and, similarly, from there via the included ratchet clutch to both wheel-tracks, and they synchronously rotate in the direction of the rotating drive shaft, leading to the straight-ahead movement of the vehicle. The turning of the vehicle is performed by turning the vertical posts of the rear axle by means of a steering device.

Both rear wheel-tracks rotate around a vertical strut of the rear axle; a shared frame (Figure 5(b)) starts to rotate and release the left or right of the ratchet clutch, and one of the wheel-tracks stops. There will be a rotation of the vehicle relative to the Instantaneous Center (IC), which will lie in the intersection of the directions of the drive shaft and the horizontal axis of the rear axle. Depending on the angle of rotational-horizontal axis of the rear axle, the position of the IC can get close to the vertical axis of the hub of the wheel-tracks and can be the same. Then, the vehicle can turn with a minimum radius towards the vertical axis of one of the wheel-tracks. The uniqueness of the vehicle is determined using the anatomical formula (structural) of the structure. The total number of independent elements of the chassis is four, namely a front driving axle, total frame, a cross-shaped rear axle, and the two rear wheel-tracks (Figure 5). Then, the number of all elements (links) is $n=5$.

The number of one-directional compounds of these elements with respect to $P_{1}$ is four, namely the junction of the common frame, (Figure 5(b)) with the drive shaft of the front axle, a cross-shaped rear axle with the general frame, and the connection of both rear wheel-tracks with two axles of the rear axle, that is $P_{1}=4$ (Figure 5). Temporary contacts of the wheeltracks on the surface of the support are four movable joints $P_{4}$, that is, $P_{4}=3$.

Because the contact of the leading wheel-tracks with the surface of the support is considered as one contact, they are rigidly connected to each other and have one connection with the surface of the support.

Contacts of the wheel-tracks on the surface of a support area are considered to be four movable parts, since the wheel-track as a separate element of the overall system has four mobilities towards the reference surface. Of the six degrees of freedom of the wheeltrack in space, there are only four free degrees, and the other two degrees of freedom are limited. As a result:

$$
n=5, \quad P_{1}=4, \quad P_{4}=3 .
$$

Then:

$$
W=6 . n-5 P_{1}-2 P_{4}=6.5-5.4-2.3=4 .
$$

The results are as follows: two movements of the slide in the directions of two mutually perpendicular directions along the axes OX and OY on the surface of the support area: one rotational movement about the axis of the drive shaft and one rotational motion around vertical axis OZ. Of the four mobility chassis relative to the reference surface, two are controlled: the straight line movement by the actuator and control by the steering wheel. The remaining two mobilities of the vehicle are not operated. Since this vehicle is programmed to be slow in speed, it makes no sense to eliminate unnecessary degrees of freedom. Received extra mobilities are the consequence of the fact that the vehicle stays on three footings when driving. Therefore, the formula considers its mobility in two planes. The transition from one plane to another is a threat at high velocities when inertial factors have a dominant influence. Chassis of modern four-wheel cars has four unmanaged mobilities. However, so far, nobody complains about this, although these mobilities are controlled by inertial factors at higher speeds and can result in tragic consequences. The scheme of the mechanism of the wheel-track contains the leading link generator made in the triangular form, and six (Figure 4(a)) support elements of equal sizes 2, 3, 4, 5, 6 , and 7 are interconnected pivotally, forming a closed, 


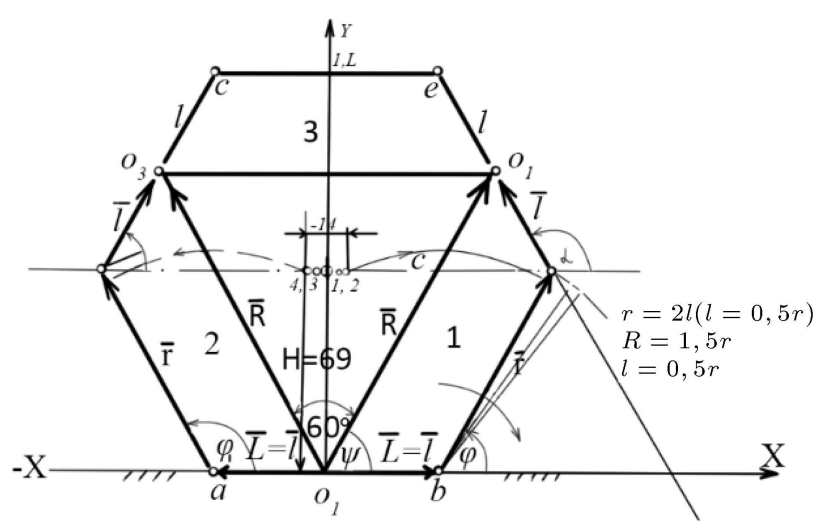

Figure 6. Kinematics of the wheel-track.

planar kinematic chain. Generator at the three corners is pivotally connected to three supporting elements of 7,3 , and 5 , forming three identically symmetrical elements placed around the generator including the hinged four-bar mechanisms (Figure 5).

The resulting mechanism consists of seven moving links $(n=7)$, the fixed link (link O) and the ten onedirection kinematic pairs $\left(P_{1}=10\right)$. The system is flat. Then:

$$
W=3 n-2 p_{1}=3.7-2.10=1 .
$$

In other words, the scheme of the mechanism has one mobility. The closed circuit (Figure 6) has:

$$
W=3 n-2 p_{1}=3.6-2.9=0,
$$

of the zero mobility.

The movement of generator uniquely determines the number of mobilities of the mechanism scheme of the wheel-track.

Figure 6 shows the kinematic chain of the wheeltracks. To determine the motion pattern of the scheme mechanism when moving one of the units (master), we need to determine the transfer function to describe the relative position of all links of the mechanism.

The mechanism of the wheel-tracks is a HighClass Mechanism (HCM). The so-called "High-Class Mechanism" can be represented as a four-bar link mechanism with a closed kinematic linkage (four links including two hinges on the supporting foot and two hinges on the triangular "wheel", and all of the "caterpillars" of the wheel rim - closed kinematic relationship). While this is a many-hinged mechanism, it is appropriate to refer to the well-known theory of manyhinged mechanisms. Approximate or approximating synthesis of hinge mechanisms originates in the works of P.L. Chebyshev. In the analysis and synthesis of articulated mechanisms, Chebyshev [22] established a mathematical apparatus of the best approximation of functions, which found wide applications in many branches of mathematics, physics, theory of electrical circuits, etc. Created by Chebyshev, methods of synthesis of mechanisms on the basis of approximating functions were further developed in the classical works of Bloch (1943) [23] and Levitsky (1948) [24]. A great contribution to the development of methods of approximate synthesis of articulated mechanisms was made by Freudenstein (1955) [25], who on the basis of a simple system of equations developed effective ways for an accurate and approximate solution to the classical problem of kinematics of flat hinge four-link chains. In [26], the method of Freudenstein or the method of displacement equations was generalized and applied to other types of planar mechanisms, in particular for a crank-driven slide mechanism. The method was also extended to spatial four-link chains. To illustrate the method, we focused on the synthesis of four-link chains to reproduce functions of the form:

$$
f(x)=\log (x) \text { and } f(x)=\frac{1}{x} .
$$

In [27], to find an approximate solution to the system of equations of Freudenstein, an efficient algorithm was proposed based on the application of orthogonalization of matrices, method of least squares. This algorithm is well suited to implement since rather complex manyhinged mechanisms are relatively easy to calculate for an arbitrary playback function. In this work, the method for the calculation by the many-hinged mechanism goes back to the solution to the classical problem of Freudenstein; however, it is original in the methodology. Further, the math for a simple case of wheel-tracks is described in detail, consisting of six reference surfaces. It is easy to show that the number of bearing surfaces can increase, which may be important for some particular cases; however, it is clear that the mathematical description of such manyhinged mechanisms will be more complicated than the simplest case with six bearing surfaces. From Figure 7, it is seen that one leading link generator $\left(O_{1}, O_{2}\right.$, or

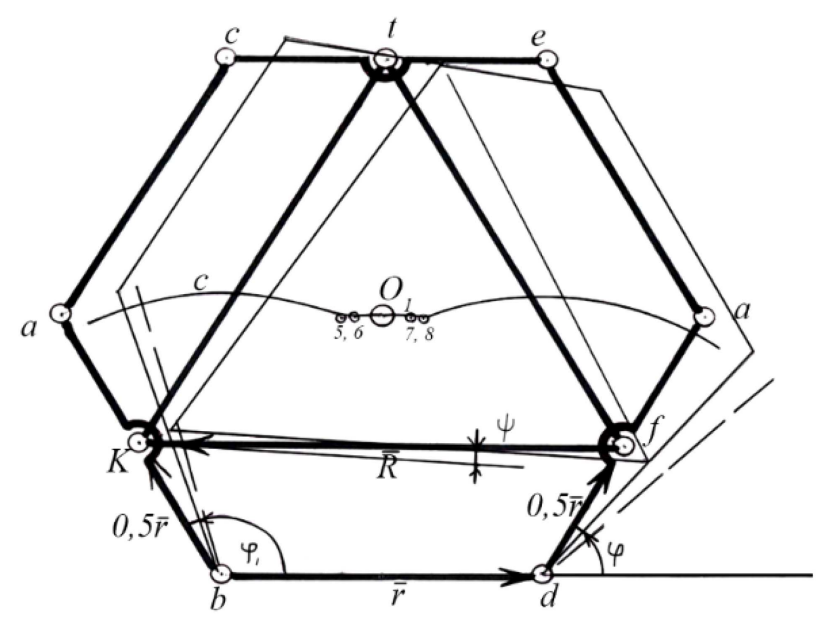

Figure 7. The kinematic calculation. 
$\mathrm{O}_{3}$ ) during rotation towards the center hub $O$ sets in motion three four-link mechanisms at the same time. Although all the units of the four-bar linkage are the same, the relevant lengths of the links have the same length:

$$
l=\frac{r}{2}, \quad R=1,5 r \quad \text { and } \quad L=l=\frac{r}{2} .
$$

In some sections, the relative motion is impeded. When the stationary (mundane) position of link ac, in Figure 7, is at focus, the center of the wheel hub $O$ can move from Positions 1 and 2 to the right and can make the same movement to the left, when the direction of rotation of the leading link changes, i.e., it has limited mobility. When the scheme wedged becomes rigid in the extreme positions, the motion of the center of the hub continues, revolving either point "c" or point "a" of a circuit. Then, it lands on another side of the hexagonal track, and the center hub will similarly start to move to the new base. Although the trajectory of the hub $O_{12}$ in the stillness of the grounds is small, it very well softens the movement of the hub, because it is caused by the interaction of three four-bar chains. At this point, the basis is not valid by a tangent component of the driving force of the actuator. On the pliable support surface, this motion effect provides extremely high smoothness, because the reproducible function of the whole complex system of conjugate fourbar linkage and the kinematic connection of the wheel rim of the caterpillar is a straight line parallel to the surface. However, it is worth emphasizing that this condition exists on a malleable surface, as it requires a certain shift of the edges of the bearing surfaces below the level of the supporting ground surface. This circumstance may cause unwanted vibration while driving on smooth and hard surfaces at high speeds, i.e., may be negative. However, movements with low speed on a pliable surface are non-negative phenomena, and, even, the penetration of the edges of the bearing surfaces into the loose soil will contribute to increasing the traction of the moving crew with the surface, on which it is moving. If the movement on the slope or terrain feature is assumed, that is very positive. When moving from one base to another base, a sharp angle "c" (Figure 7) of the hexagon deepens to a pliable surface. This creates favorable conditions; on the one side, it eliminates the slipping of the wheel-track on the supporting surface; on the other side, it reduces the height of the trajectory "C" of the hub at this moment, bringing it closer to straightness. To determine the characteristics of the mechanism scheme analytically, the formula of the kinematic model of the scheme is required. Let us try defining this function. We should replace closed contours 1 and 2 in Figure 7 with the vectors $\bar{r}, \bar{l}$, and $\bar{R}$ in the following way:

$$
\bar{l}+\bar{r}+\bar{l}=\bar{R} \text {. }
$$

Let us represent it on the coordinate axes $O, X$, and $Y$, and we will get the following:

$$
\begin{aligned}
& l+r \cos \varphi+l \cos \alpha=R \cos \Psi, \\
& r \sin \varphi+l \sin \alpha=R \sin \Psi .
\end{aligned}
$$

As $l=0,5 r$ and $R=1,5 r$, let us insert them:

$$
\left\{\begin{array}{l}
0,5 r+r \cos \varphi+r \cos \alpha=1,5 r \cos \varphi \\
r \sin \varphi+r \sin \alpha=1,5 r \sin \varphi
\end{array}\right.
$$

Different step values for angle $\varphi$ are set, given that $\varphi$ changes starting from $60^{\circ}$. Let us square the system of Eqs. (8) and sum them up. After conversion, we will get the following:

$$
r^{2} \cos \varphi+2 r^{2}(0,5+\cos \varphi) \cdot \cos \alpha=2 r^{2} \sin \varphi \cdot \sin \alpha .
$$

Let us denote the constants using:

$$
A=r^{2} \cos \varphi, B=r^{2}+2 A, C=2 r^{2} \sin \varphi,
$$

and we get:

$$
A+\left(r^{2}+A\right) \cos \alpha=-C \sin \alpha,
$$

or:

$$
A+\left(r^{2}+A\right) \cos \alpha=-C \cdot \sqrt{1-\cos ^{2} \alpha} .
$$

Let us get rid of the square root:

$$
\begin{gathered}
{\left[\left(r^{2}+A\right)^{2}+C^{2}\right] \cos ^{2} \alpha+2 A(r+A) \cos \alpha} \\
+A^{2}-C^{2}=0 .
\end{gathered}
$$

Let us denote the constants:

$$
\begin{aligned}
& D=\left[\left(r^{2}+A\right)^{2}+C^{2}\right], \\
& E=2 A\left(r^{2}+A\right),
\end{aligned}
$$

and we will get:

$$
\cos \alpha=\frac{E}{D} \pm \sqrt{\frac{E^{2}}{D^{2}}-\frac{A^{2}-C^{2}}{D}}=0,
$$

or:

$$
\alpha=\arccos \left(-\frac{E}{D} \pm \sqrt{\frac{E^{2}}{D^{2}}-\frac{A^{2}-C^{2}}{D}}\right) .
$$

From Eq. (9), we will get:

$$
\Psi=\arcsin \left(\frac{r \sin \varphi+r \sin \alpha}{1,5 r}\right) .
$$


Further, for Circuit 2 (see Figure 7), similarly, we get the system of equations:

$$
\left\{\begin{array}{l}
r\left(0,5+\cos \varphi_{1}\right)+r \cos \alpha_{1}=1,5 r \cos \left(\Psi+60^{\circ}\right) \\
r \sin \varphi_{1}+r \sin \alpha_{1}=1,5 r \sin \left(\Psi+60^{\circ}\right)
\end{array}\right.
$$

From the obtained values of $\boldsymbol{\Psi}$, we will determine $\alpha_{1}$ and $\varphi_{1}$ :

$$
a_{1}=\arccos \left(-\frac{E_{1}}{D_{1}} \pm \sqrt{\frac{E_{1}^{2}}{D_{1}^{2}}-\frac{A_{1}^{2}+C_{1}^{2}}{D}}\right) .
$$

From Eq. (11), we will get:

$$
\sin \varphi_{1}=1,5 \sin \left(\Psi+60^{\circ}\right)-\sin \alpha,
$$

in which:

$$
\phi_{1}=\arcsin \left(1,5 \sin \left(\Psi+60^{\circ}\right)-\sin \alpha_{1}\right) \text {. }
$$

Using Eqs. (26), (27), (28), and (30), we plot the points "c" and "e" of the mechanism scheme (see Figure 7 ).

If the length of the connecting link of the "c e" is equal to a given length, $r$, of the sides of the hexagon, i.e.:

$$
\mathrm{ce}=\mathrm{ac}
$$

then the scheme of the mechanism exists, and we continue to determine the following values of the angle of the leading link:

$$
\varphi_{1}=\varphi+\Delta .
$$

$\varphi=60^{\circ}$ is the initial value and, then, it decreases upon the increments of $\Delta$ given that the found value will not diverge, i.e.:

$$
\text { ce } \neq r \text {. }
$$

At the moment when $c e \neq r$, the scheme of the mechanism is wedged. Further rotation of the generator will cause the rotation of scheme as a rigid system towards the point "c", and the scheme will establish the following ground (Figure 7). The first scheme position differs from the second position (Figure 7 ), because, in the first case, the generator makes a rotational movement towards the center $O_{1}$ and, in the second case, it makes a complicated plane-parallel movement as the connecting rod. In the first case, the trajectory of the hub $O_{12}$ is the arc of a circle; in addition, Positions 5, 6, $O_{1}, 7$, and 8 shown in Figure 7 are parts of the connecting rod curve. Therefore, the second stage of the analysis will start with an analysis of the position of the hinge of four-bar linkage $\alpha f k B(\bar{r}, 0,5 \bar{r}, \bar{R}, 0$, and $5 \bar{r})$, i.e., with the analysis of:

$$
\bar{r}+0,5 \bar{r}+\bar{R}=0,5 \bar{r},
$$

that is:

$$
\left\{\begin{array}{l}
r+0,5 r \cos \varphi+1,5 r \cos \Psi=0,5 r \cos \varphi_{1} \\
0,5 r \sin \varphi+1,5 r \sin \Psi=0,5 r \sin \varphi_{1}
\end{array}\right.
$$

From this system, we can determine the angles $\Psi$ and $\varphi_{1}$ as the positions of the scheme points a and $\mathrm{a}_{1}$.

Next, the positions of the points "e" and "c" are determined, which must lie in the same straight line with point $t$, the top of the generator, shown in Figure 7. Otherwise, the calculations are repeated reciprocally to determine the measured value of the angle $\varphi$. Thus, by a two-stage calculation, the positions of the scheme of the mechanism of the wheel-tracks can be determined. For the following positions, the calculations of the first stage are used again. As can be seen from the algorithm, the calculations involving computing and building the positions of the mechanism scheme can be performed using modern computers.

\section{Conclusion}

This study aimed to combine through one mechanism the advantages of walking-type travel and the wheels so as to create the concept of the wheel-tracks. The proposed design of the wheel-track was subjected to mathematical modeling, and the result of mathematical modeling was the conclusion about the suitability of the playback features of the complex multi-link mechanism, wheels, and tracks to ensure smooth motion without slip of a vehicle on a loose surface. This work demonstrated the applicability of the proposed approach to the design of vehicles that were forced to move along a strong off-road. This paper demonstrated the superiority of the proposed approach in terms of requirements to unmanned exploratory machines and robotized platforms that are compelled to act in the aftermath of natural and man-made disasters.

\section{References}

1. El-Gawwad, K.A., Crolla, D.A., Soliman, A.M.A., and El-Sayed, F.M. "Off-road tyre modelling III: effect of angled lugs on tyre performance1", Journal of Terramechanics, 36(2), pp. 63-75 (1999).

2. Abdelrahman, M., Zeidis, I., Bondarev, O., Adamov, B., Becker, F., and Zimmermann, K. "A description of the dynamics of a four-wheel mecanum mobile system as a basis for a platform concept for special purpose vehicles for disabled person", in Shaping the Future by Engineering: 58th Ilmenau Scientific Colloquium, Technische Universität Ilmenau (2014).

3. Giurgiu, T., Puică, C., Pupăză, C., Nicolescu, F.A., and Zapciu, M. "Mecanum wheel modeling for studying roller-ground contact issues", U.P.B. Sci. Bull., Series D, 79(2), pp. 147-158 (2017).

4. Surovec, R., Gmiterko, A., Vacková, M., Virgala, I., Prada, E., and Pipík, T. "Design of robot vehicle un- 
dercarriage with ability to operate in broken terrain", Procedia Engineering, 48, pp. 650-655 (2012).

5. Harrington, B.D. and Voorhees, C. "The challenges of designing the rocker-bogie suspension for the mars exploration rover", in 37th Aerospace Mechanisms Symposium, Houston, TX, United States (2004).

6. Malenkov, M.I., Volov, V.A., Guseva, N.K., and Lazarev, E.A. "Increasing the mobility of Mars rovers by improving the locomotion systems and their control algorithms", Russian Engineering Research, 35(11), pp. 824-831 (2015).

7. Kim, Y., Eom, W., Lee, J.H., and Sim, E.S. "Design of mobility system for ground model of planetary exploration rover", Journal of Astronomy and Space Sciences, 29(4), pp. 413-422 (2012).

8. http://www.yankodesign.com/2009/03/10/split-yourwheel-into-eight/

9. http://www.michelinchallengedesign.com/thechallenge-archives/2009-brave-bold/2009-showcase-ofselected-entrants/transforming-multifunctionalwheels-by-su-yan g-park-and-chang-hoe-heo-southkorea/.

10. Muratov, A.M., Omarov, A.D., Kaynarbekov, A.K., and Sazanbaeva, R.I. "Synthesis of the scheme of the walking wheel", p. 227, Bastau, Almaty (2013).

11. Muratov, A.M. and Kaynarbekov, R.I., Walking Drivers, p. 182, Bastau, Almaty: Textbook (2000).

12. Muratov, A.M. and Sazanbaeva, R.I., Improving the Patency of Wheeled Vehicles in Off-Road Conditions, Bastau, Almaty: Textbook (2003).

13. Muratov, A.M. and Kaynarbekov, A.K. "Trackwalking propulsion of the vehicle, provisional patent RK No11006, 14.11.2001", in Bulletin No 12, Almaty (2001).

14. Omarov, A.D., Muratov, A.M., Kaynarbekov, A., and Bekmambeth, K.M. "Off-road vehicles", Patent of Kazakh Republic, No. 12-3/3205, p. 182, Almaty (2014).

15. Briskin, E.S., Zhoga, V.V., Chernyshev, V.V., and Maloletov, A.V. "Bases of calculation and designing of walking machine with cyclic vehicles", in Monograph, p. 164, Moscow, Mashinostroenie (2006).

16. Omarov, A.D., Muratov, A., Kaynarbekov, A., and Bekmambeth, K. "Vehicles to drive on extra complicated support surface (design and calculations)", in LLP, Alla Prima, Textbook, p. 118, Almaty (2016).

17. Muratov, A., Kaynarbekov, A., and Bekmambeth, K. "Walking wheel for terrestrial vehicle. Materials of XII international scientific and practice conference", Modern European Science, Chemistry and Chemical Technology Mathematics Technical Science, 9, pp. 5864 (2016).
18. Muratov, A. and Kaynarbekov, A. "Walking wheel for vehicles. Materials of XII international scientific and practice conference", Proceedings of Academic Science, 4, pp. 75-78 (2016).

19. Omarov, A.D., Muratov, A., and Kaynarbekov, A. "The anatomical structure and the kinematic model the wheel - tracks of all-terrain vehicles", Industrial Vehicles of Kazakhstan, Almaty, 3(52), pp. 18-23 (2016).

20. Kaynarbekov, A. and Taninbergenov, A.K. "Walking wheel $<<$ TUK - TUK $>>$ ", Collection of Scientific Works of Ukrainian State Academy of Railway Transport, Kharkov, Ukraine, 148(1), pp. 164-170 (2014).

21. Kaynarbekov, A., Zhumabek, A.G., and Omarova, G.A. "The elimination of the defect of the gait of a walking wheel for mounting to vehicles", Monthly Scientific Journal, 2(3), pp. 24-27 (2014).

22. Chebyshev, P.L., Théorie des Mécanismes Connus Sous le Nom de Parallé Logrammes, Imprimerie de l'Académie Impériale des Sciences (1853).

23. Bloch, Z.Sh. and Karpin, E.B., Practical Methods for the Synthesis of Planar Four-Link Mechanisms, Publishing house of AS SSSR (1943).

24. Levitsky, N.I. "Application of quadratic approximation of functions to the solution of problems of synthesis of mechanisms" in, Proceedings of the Seminar on TMM, Publishing house of AS SSSR, 17 (1948).

25. Freudenstein, F. "Approximate synthesis of four-bar linkages", Transactions of ASME, 77, pp. 853-861 (1955).

26. Hartenberg, R.S. and Danavit, J., Kinematic Synthesis of Linkages, McGraw-Hill, New York (1964).

27. Liu, Z. and Angeles, J. "Least-square optimization of planar and spherical flour-bar function generator under mobility constraints", Journal of Mechanical Design, December, 114, pp. 569-573 (1992).

\section{Biographies}

Abil M. Muratova works at Kazakh University of Railway Transport and is a Professor at the Transport Technology, Mechanical Engineering and Standardization Department, Almaty, Kazakhstan. She has over 350 scientific papers, including recourse books and study guides.

Assemkhan K. Kaynarbekov works at Kazakh University of Railway Transport and is an Associate Professor at the Radio Engineering, Electronics and Telecommunications Department, Almaty, Kazakhstan. He has over 200 scientific papers including recourse books and study guides.

Suleimen K. Sultangazinov works at Kazakh University of Railway Transport and is the Head of the Automation and Transport Information Systems 
Department, Almaty, Kazakhstan. He has over 170 scientific papers, including recourse books and study guides.

Taizhan S. Sarzhanov works at Kazakh University of Railway Transport and is the Head of the Postgraduate Study Department, Almaty, Kazakhstan. He has over 100 scientific papers, including recourse books and study guides.

Aspan K. Kazhigulov received DSc in Engineering. He works at Kazakh University of Railway Transport and is an Acting Professor at the corresponding Department. He has over 100 scientific papers, including recourse books and study guides.

Abdiashim Shalkarov is an Associate Professor at Kazakh University of Railway Transport. He has over 80 scientific publications.

Gulmira S. Mussaeva works at Kazakh University of Railway Transport and is a Professor at the Transport
Construction Department. She has over 100 scientific papers, including recourse books and study guides.

Kanagat M. Bekmambet works at Kazakh University of Railway Transport and is an Associate Professor at the Transport Technology, Mechanical Engineering and Standardization Department. He has over 52 scientific papers, including recourse books and study guides.

Bekture Sh. Yessengarayev has a $\mathrm{PhD}$ degree in Engineering. He works as the Head Manager at the Labor Safety and Ecology Department of the JSC, "Kazakhstan Temir Zholy" branch, "Directorate of Trunk Network". He has over 20 scientific papers, including recourse books and study guides.

Amangeldi Tanirbergenov works as an Associate Professor at Railway Track, Railway Location Survey and Design Department, Kazakh University of Railway Transport. He has over 60 scientific papers, including recourse books and study guides. 